\title{
Nanocarbon powder for latent fingermark development: a green chemistry approach
}

\author{
Revathi Rajan ${ }^{1}$, Yusmazura Zakaria², Shaharum Shamsuddin² and Nik Fakhuruddin Nik Hassan ${ }^{1 *}$ (B)
}

\begin{abstract}
Background: Optimal fingermark developing product would have the following attributes; small, rounded and well-dispersed particles, which has high affinity for the fingermark residue but exhibits minimal interaction with the substrate bearing the fingermark. However, most commercial powder formulations still utilise fine particles in the range of 1 to $10 \mu \mathrm{m}$.

Results: Ageing of the filtrate from rice husk acid digestion produced amorphous nanocarbon powder (NP) that exhibited non-smooth, slightly irregular spherical particles with a higher degree of agglomeration. Particle size distribution fell in the range of 100 to $500 \mathrm{~nm}$ with mean particle size of $300 \mathrm{~nm}$. Molecular bonding and chemical composition of the NP was characterised using spectroscopic techniques. Findings revealed that the nanoparticle powders and reagent performed on par with the existing commercial powders and exhibited higher selectivity. Nanoparticle synthesis from low-cost precursor in this research did not require special equipment, addition of resins or adhesives, or surface passivation.
\end{abstract}

Conclusion: Nanocarbon powder is exploitable as fingermark developing powder generated from a sustainable source.

Keywords: Carbon nanoparticle, Nanocarbon powder, Rice husk, Latent fingermark, Visualisation

\section{Background}

Dactylography, the study of fingerprints as a means of identification is one of the fundamental science in criminal investigations (Nithin et al. 2009). Most of the time, these fingermarks are invisible and require physical or chemical reagents for visualisation (Champod et al. 2004; Jelly et al. 2009). Choice of the technique used for fingermark development relies on factors such as surface porosity and smoothness, the age of fingermark, the composition of fingermark and the recovery conditions (Sears et al.; 2012; Dhall et al. 2016). Powdering technique is the most basic and common approach for fingermark development in the crime scene (Becue et al. 2011; Yamashita and French 2011). Powder particles adhere to the moist and oily component of the fingermark residue (Champod et al. 2004; Choi et al. 2008). The size, shape and agglomeration state of the powder particles play a large part in determining the amount of adhesion they have to the fingermark (Wilshire, 1996; Chadwick et al.

\footnotetext{
* Correspondence: nikf@usm.my

${ }^{1}$ Forensic Science Programme, School of Health Sciences, Universiti Sains

Malaysia, Health Campus, 16150 Kubang Kerian, Kelantan, Malaysia

Full list of author information is available at the end of the article
}

2012). Fine and rounded particulates adhere better than large, coarse ones. Hence, most powder formulations are composed of particles of size ranging between 1 and $10 \mu \mathrm{m}$ (Wilshire, 1996; Sodhi and Kaur, 2001). Commercial black powder commonly contains black iron oxide, rosin, lamp black, fuller's earth and manganese dioxide which acts as colourant as well as adhesive substance that increases adherence (Goode and Morris, 1983; Lee and Gaensslen, 2012).

Recent innovations in nanotechnology enable fabrication of nanoparticles that have desired shape, size and agglomeration. Efforts to synthesise novel nanoparticle are still being carried out despite abundant discoveries mainly to improve physical properties and chemical functionality of the nanoparticle for diversifying its uses. It is imperative not only to synthesise new nanoparticle that is sustainable, non-toxic and biocompatible but also to adopt a green synthesis process using renewable precursors (Rajan et al. 2018).

Agricultural wastes, such as rice husk and bamboo leaves have proven to be excellent sources for nanoparticle extraction (Wang et al. 2010; Vaibhav et al. 2015; Lee et al. 2017). Rice husk, a byproduct of rice milling generated in billions 
of tones yearly, acts as a source of nanocellulose, nanosilica and nanocarbon (Luduena et al. 2011; Liu et al. 2012). Carbon exists naturally as a black material with poor water solubility and weak fluorescence (Wang and HU, 2014). However, nanosized carbon has demonstrated to be a viable alternative to conventional quantum dots due to integral advantages such as bright fluorescence, high photostability as well as tunable excitation and emission spectra (Han, 2009; Roshni and Ottoor, 2015). Carbon nanoparticles also exhibit less cytotoxicity, solubility in water, chemical inertness and have good biocompatibility as previously reported (Peng and Travas, 2009; Ngu et al. 2016). Intrinsic properties of carbon nanoparticles can be finely tuned by altering morphological and structural parameters leading to various research in synthesising micro and nanospheres of carbon (Garrigue et al. 2004; Sun and Li, 2004).

Carbon nanoparticle has numerous intriguing and novel applications in electronics, chemistry, biology and material sciences (Branca et al. 2004). It can be used as pigments, fillers, supercapacitors, bioimaging dye, photocatalysis, optoelectronics, drug delivery, biodiagnostics, high-performance electrode material in batteries and optical sensing (Meiling et al. 2016; Shen, 2017). Carbon can also be utilised in the form of activated carbon for water treatment, catalyst carriers, electrode materials and for hydrogen storage. Synthesis of carbon nanoparticle usually uses coal as a precursor in industrial sector but biomass such shells, cork powder, wood, bamboo and herb residue can be converted into activated carbon (Song et al. 2012).

Carbon nanoparticle can be manufactured through top-down or bottom-up approaches (Balasooriya et al. 2017). Top-down industrial synthesis of carbon nanoparticle is usually carried out through laser ablation of graphite (Sun et al. 2006; Cao et al. 2007), electrochemical oxidation of graphite and multiwalled carbon nanotubes (Zhao et al. 2008; Hu et al. 2009), chemical oxidation of arc-discharged single-walled carbon nanotubes or candle soot, proton beam irradiation of nanodiamonds or in other words breaking down large molecules (Ray et al. 2009; Zhu et al. 2009; Li et al. 2011a, b). Alternatively, carbon nanoparticle can also be synthesised through bottom-up approach involving the synthesis of atoms and subsequent polymerisation. Bottom-up techniques include pyrolysis, microwave plasma-enhanced chemical vapour deposition, electrolysis of molten salt, graphitisation of particles obtained by microemulsion polymerisation and treatment of supercritical water (Garrigue et al. 2004).

Industrial top-down approaches pose many disadvantages when manufacturing was made in bulk quantities although the techniques are state of the art. Processes were energy intensive used up fossil fuels simultaneously generated greenhouse gases, involved complex processes with severe synthetic conditions and required costly precursors such as graphite (Zhu et al. 2009). Bottom-up approaches have their own disadvantages such as energy consuming at a larger scale, required acidic molecular precursors as well as specific and expensive equipment. In addition, most methods reported large size distribution range that hampered efficient emission (Fan et al. 2014).

Energy shortage and environmental crisis have been an ever-growing concern, demanding facile, low-cost, sustainable and environmentally non-toxic routes of carbon nanoparticle production that can sufficiently meet customer demands ( $\mathrm{Hu}$ et al. 2010). In this instance, biomass comes forth as an excellent alternative precursor offering high-quality sustainable source. Numerous studies have utilised natural resources such as glucose, sucrose, citric acid, pomelo peel, willow bark, oatmeal, rice husk and honey to produce carbon nanoparticle (Wu et al. 2013; Shen et al. 2014; Van et al. 2014; Yu et al. 2015).

Previous research indicated that nanocarbon powder (NP) obtained from acid digestion of rice husk displayed the potential of developing latent fingermark on light background (Rajan et al. 2018). Hence, the aim of the present study was to further determine the effectiveness of NP fabricated from rice husk for the development of latent fingermark. This study was not only conducted to incorporate green chemistry into forensic field, but also to determine the effect of using nanopowder synthesised with specific characteristics that complements its use as fingermark developing powder.

\section{Methodology \\ Preparation of NP}

Details of NP fabrication from rice husk have been previously reported (Rajan et al. 2018). Nanocarbon particle was formed by ageing the filtrate from acid digestion of rice husk at $90^{\circ} \mathrm{C}$ for $18 \mathrm{~h}$. Purified NP pellet was acquired by repeated washing, and the black pellet obtained from high-speed centrifugation was subjected to drying using alcohol dehydration. Fabricated NP was characterised using several techniques such as Fourier transform infrared (FTIR), X-ray diffraction (XRD) and energy dispersive (EDX) spectroscopy as well as scanning electron microscopy (SEM).

\section{The development of fingermarks using NP}

Sixteen substrates were chosen for the fingermark experiments. These includes non-porous surfaces, such as CD-ROM, phone screen protector, gloss painted wood, stainless steel tool, electrical switch and porcelain crucible, and semi-porous surfaces, such as credit card, brown tape, currency note and translucent plastic.

To establish the details of interaction of the NP with the fingermark constituents, sebaceous, eccrine and natural fingermark obtained from five donors were developed using NP. These tests were conducted on porcelain 
crucible, in tandem with commercial black powder (BP) to compare the interaction of both powders.

To determine the sensitivity of the NP, three phase of tests were conducted; multiple donor, depletion and ageing studies. Multiple donor study was conducted by acquiring natural fingermark deposited on porcelain crucible from 36 random subjects. Depletion study was conducted by depositing 10 consecutive fingermarks on each surface. In both multiple donor and depletion studies, fingermarks were split in two halves (by applying two porcelain crucibles) and each halve developed using nanocarbon and commercial black powder, respectively. Whole fingermarks deposited on clean surfaces were weathered/aged in hot air oven to simulate the environmental exposure within a shorter time frame. Ageing was conducted at $50{ }^{\circ} \mathrm{C}$ and $100{ }^{\circ} \mathrm{C}$ at 1,2 and 3-h intervals to simulate exposure to medium and high temperatures such as in the heated confined environment. Depletion and multiple donor studies were conducted in triplicates to minimise external factors. All the fingermark samples were left open to the air for an hour before developing.

The powders had been applied by using the SIRCHIE squirrel hair brush using the powder dusting technique. Images of the fingermarks were snapshot with Canon Powershot SX60 HS with 16.1 MP resolution. After that, the fingermarks were graded/scored via scale, as presented in Table 1 (Fieldhouse et al. 2016). The recorded scores derived from NP and BP efficiency tests were analysed with Wilcoxon signed-rank test in order to identify the significant variances between the tested powders. The statistical analyses were carried out using IBM SPSS Statistics 22.

\section{Results and discussion}

\section{Characterisation of fabricated NP}

The NP that had been synthesised appeared to be extremely fine. As such, an investigation of the nanostructures of NP and BP was performed via SEM to determine the variance of particles in these two powders. Figure 1 illustrates image of NP and BP particles derived from SEM, whereby the particle sizes were $\sim 300 \mathrm{~nm}$

Table 1 Fingermark grading scale system

\begin{tabular}{ll}
\hline Grade & Description \\
\hline 0 & Fully smudged outline of a mark or no evidence of mark \\
1 & Presence of several ridges and cannot lead to identification \\
2 & $\begin{array}{l}\text { Major part of the mark is smudged, several ridge details are } \\
\text { present and analysis cannot be performed }\end{array}$ \\
3 & $\begin{array}{l}\text { Minor part of mark is smudged, most ridge details are visible } \\
\text { and analysis can be performed }\end{array}$ \\
4 & $\begin{array}{l}\text { Full mark and ridge details are clearly visible, some ridge lines } \\
\text { maybe thinned or smudged but identifiable mark }\end{array}$ \\
5 & Full mark and all ridge details are clearly visible; identifiable mark \\
\hline
\end{tabular}

and $\sim 100 \mathrm{~nm}$ for NP and BP, respectively. NP particles appeared more rounded and BP particle possessed cubical structure. Increment in surface area was generated by the reduction in particle size, which increased the amount of molecule binding to the fingermark.

The elemental analysis that was performed via EDX exhibited the existence of carbon and oxygen at an approximate atomic ratio of 7 to 3 . In addition, the absence of any other kind of elements ratifies the aspect of purity for the powder. Infrared spectrum revealed peaks characteristic of $\mathrm{C}-\mathrm{OH}, \mathrm{C}-\mathrm{H}$ and $\mathrm{C}=\mathrm{O}$ at 3420,2934 and $1613 \mathrm{~cm}^{-}$ ${ }^{1}$ respectively (Li et al. 2011a, b). The peak at 1384 and $1265 \mathrm{~cm}^{-1}$ was assigned to symmetric/asymmetric vibrations of carboxylate groups (Wu et al. 2013). It is evident that the surface of NP as indicated in the IR spectrum was rich with hydrophilic group consistent with previous reports (Xu et al. 2004). XRD analysis confirms the amorphous nature of the NP, with the absence of any sharp peaks corresponding with crystalline structure.

\section{Powder selectivity}

Figure 2 illustrates the eccrine, the sebaceous and the normal fingermarks obtained from three volunteers and developed with NP and BP. Although, true sebaceous fingermark would be unlikely to be produced, and it must be recognised in reality, sebaceous fingermark will contain eccrine components due to the ubiquitous nature of eccrine glands. According to Sametband et al. (2007), sebaceous fingermarks generally displayed a higher contrast than those of eccrine and natural origin, due to the higher concentration of moisture and grease in the sebaceous secretions. The outcomes displayed that latent fingermarks derived from varied secretions offered differing degrees of clarity, and some samples indicated discernible patterns for the ridges. As shown in Fig. 2, the contrast of natural fingermarks developed using NP was low in comparison to BP, but appeared with better contrast in the sebaceous fingermarks.

Fingermarks possess varying elements mainly because of the differing skin secretions. The sebaceous fingermarks displayed discernible ridge details as compared to the natural and eccrine fingermarks. As such, interaction occurred, primarily, between the greasy element in fingermarks and moisture. The findings also demonstrated that fingermarks with NP had lower contrast, when compared to that developed with BP. Nevertheless, increment in selectivity was exhibited by NP to ridge residue of the fingermarks, in comparison to that of BP. In addition, the increased BP adherence causes smudging of ridge details.

Figure 3 illustrates several images derived from SEM for latent fingermarks on non-porous surface developed by using brushing technique. The fingermark residue appeared to be rich in sebaceous secretions and displayed 


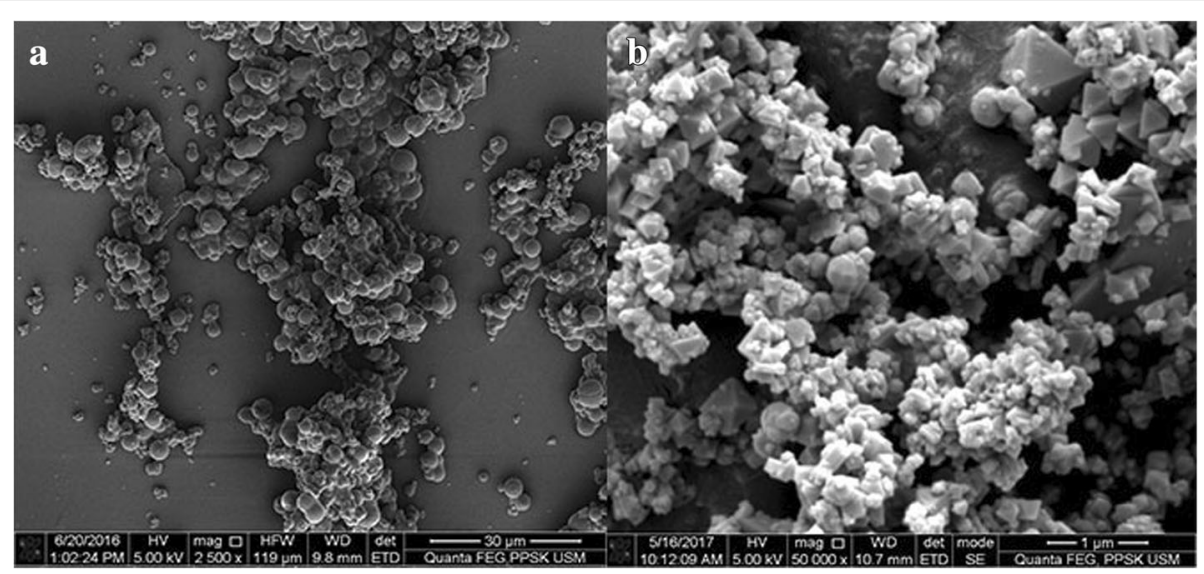

Fig. 1 SEM images of NP (a) and BP (b)

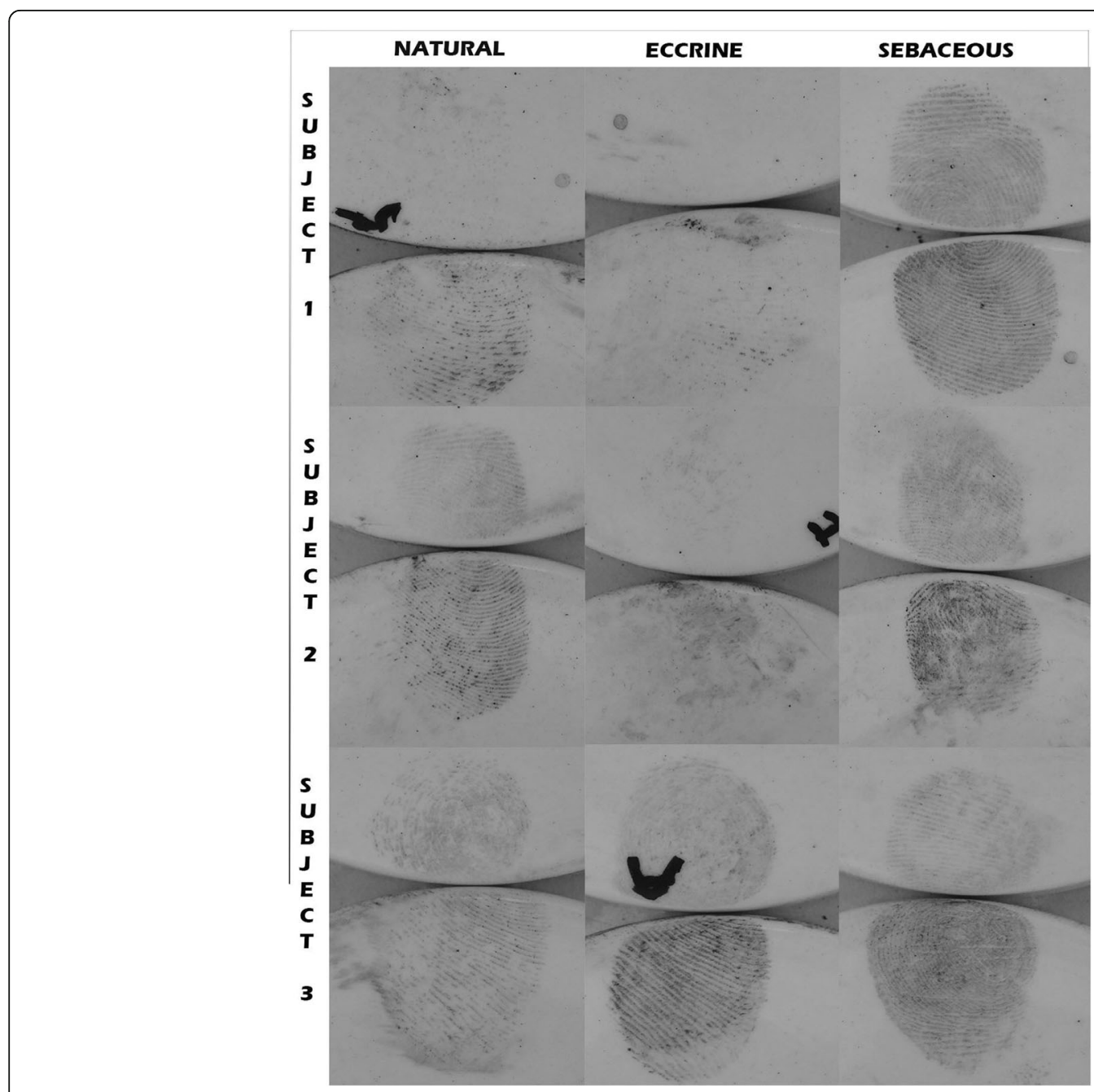

Fig. 2 Latent fingermarks of natural, eccrine and sebaceous origin from three varied subjects with upper half developed using NP, and lower half developed using BP 


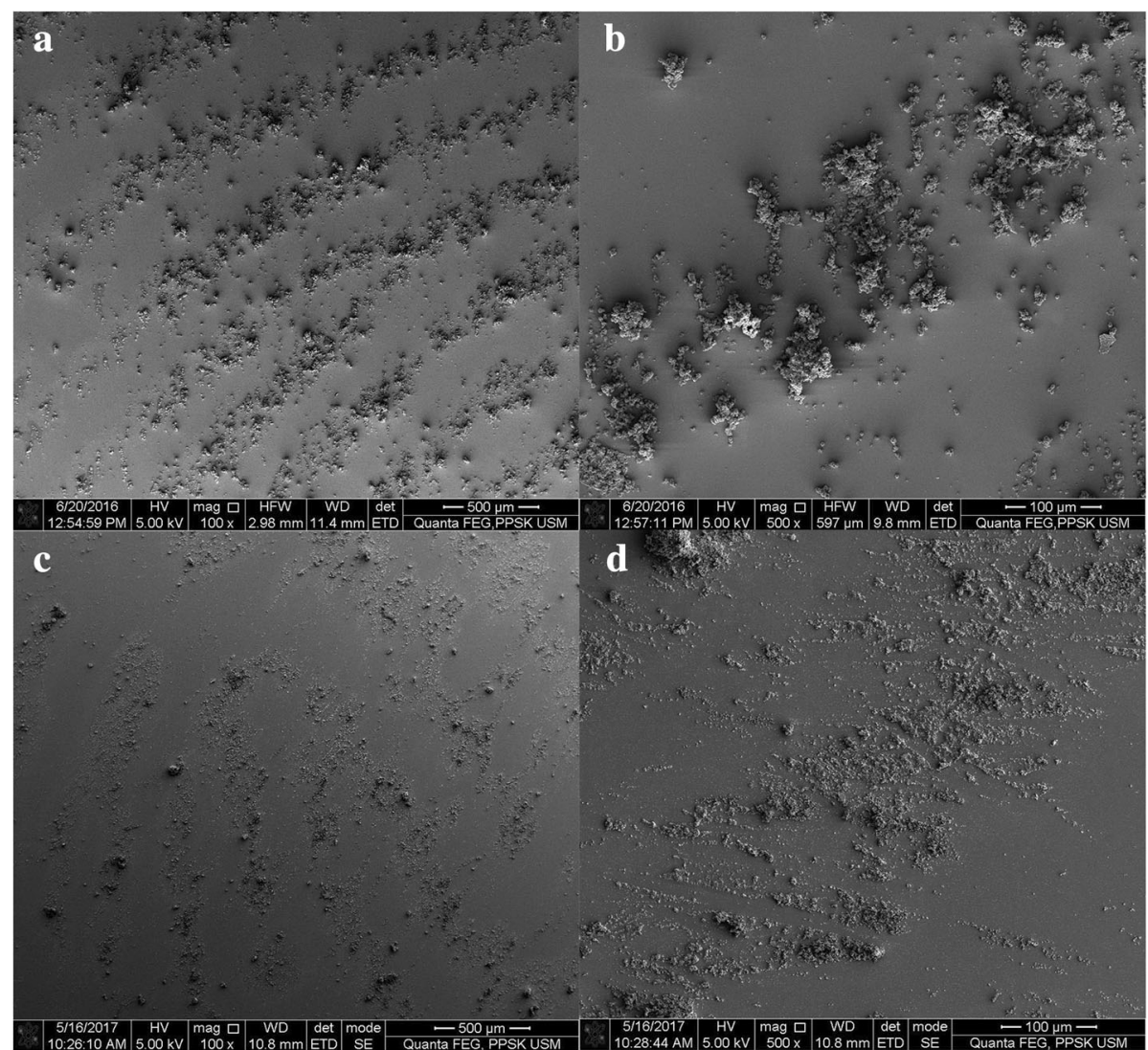

Fig. 3 SEM images of developed fingermark; NP $(\mathbf{a}, \mathbf{b})$, and BP $(\mathbf{c}, \mathbf{d})$ with natural fingermarks on glass surface

the fingermark, mainly due to the hydrophobic/non-covalent interface between $\mathrm{NP}$ and $\mathrm{BP}$ and residue of fingermarks.

The fingermark that was developed with NP displayed clear distinction between the grooves and valleys of fingermark ridges. Nevertheless, the particles of NP appeared as clusters, contributing to sporadic distribution along the fingermark ridge residue. On the other hand, latent fingermark developed with BP particles revealed extremely fine particles dispersed homogenously along the grooves and valleys of the fingermark, with slightly higher concentration particle along the fingermark grooves. In term of contrast and clarity, the NP appeared to have the upper hand in comparison to BP.

\section{Powder sensitivity}

Figure 4 illustrates the score of levels for the fingermarks presented in bar chart to ease data interpretation regarding performance of the studied powders. The multiple donor study, conducted to compare the effectiveness of $\mathrm{NP}$ and $\mathrm{BP}$ on a range of fingermarks contributed by a variety of donors, showed that the NP displayed notable superiority in performance. The average scores of NP and $\mathrm{BP}$ were 2.50 and 2.00 , respectively.
Fingermark contains many constituents due to the secretions from various skin glands and external contaminants. Figure 5 portrays example of fresh fingermarks deposited on both non-porous and semi-porous surfaces, developed with NP. Fresh fingermarks developed with NP exhibited outstanding clarity and contrast, magnifying each minute detail. Figure 6 displays fingermarks developed using NP in the ageing study at various time and temperature intervals. As anticipated, the quality of the fingermarks developed deteriorated with increase in ageing time and temperature, due to loss of moisture and absorption/dispersion of fingermark residue into the fingermark bearing substrate.

The findings of the sensitivity analysis, comprising of the depletion and ageing studies, indicated successful development of latent fingermarks on majority of the surfaces tested. Nevertheless, it was noted that the BP had exhibited better performance with the surface of porcelain crucible, while the NP worked rather well on reflective surface (e.g., CD-ROM, stainless steel), as shown in Fig. 7. NP developed fingermark had metallic brown colour in appearance. However, NP performed poorly on polymer-based products such as currency note, brown tape, credit card and translucent plastic file, after certain 


\section{Multiple donor study using natural finger-marks from 36 subjects}

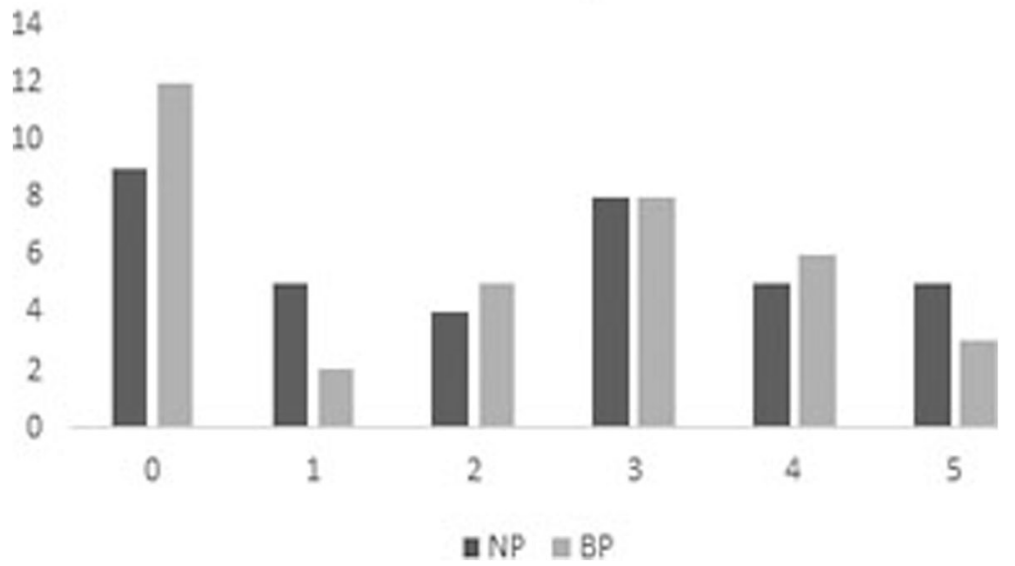

Fig. 4 Bar chart that reflects the cumulative number of fingermarks in each score category

ageing intervals. Previous study has reported that polymer-based products exhibited absorption of lipid components of fingermarks when exposed to high temperatures. Especially acrylic polyvinylchloride is an amorphous polymer, making it porous and hydrophobic, which readily absorbs the lipid residues of the fingerprint residue (Cohen et al. 2012).

The outcomes retrieved from the depletion and ageing studies for the tested surfaces are displayed in line graphs (Figs. 7 and 8) to represent non-porous and semi-porous surfaces. The line graphs indicated that the performance of NP was in line with that of BP, while better in several surfaces, except on currency note, gloss painted wood and translucent plastic (Fig. 9).

The Wilcoxon signed-rank test was carried out to compare the effectiveness displayed between NP and BP obtained from the surface test study through the use fingermark scores. Table 2 displays the outcomes of Wilcoxon signed-rank tests analysed based on fingermark grades retrieved from the surface experimental tests.

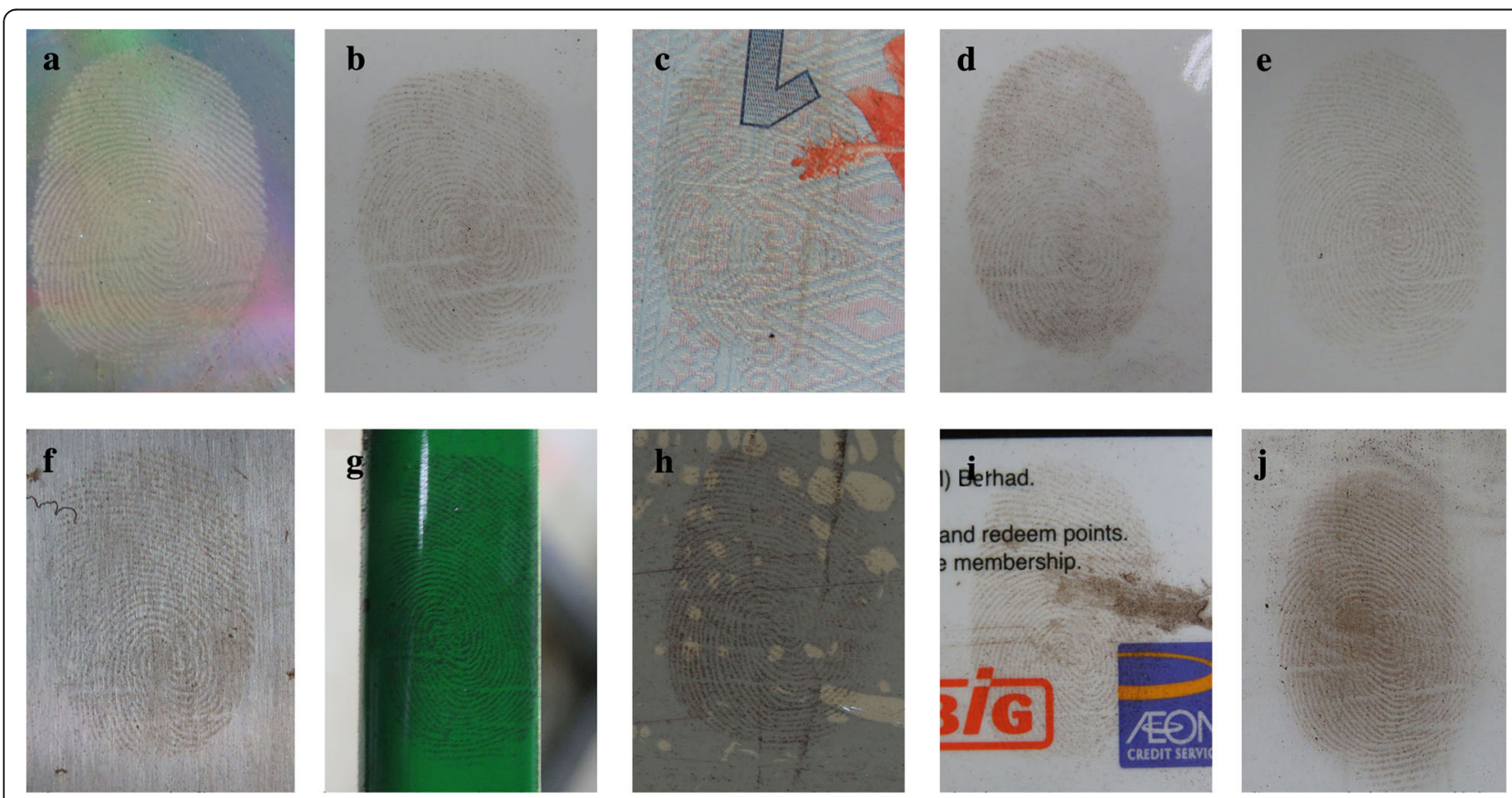

Fig. 5 Fresh fingermarks developed using NP on a CD-ROM, $\mathbf{b}$ tempered glass, c currency note, $\mathbf{d}$ porcelain crucible, e translucent plastic, $\mathbf{f}$ gloss painted wood, $\mathbf{g}$ stainless steel tool, $\mathbf{h}$ brown tape, $\mathbf{i}$ credit card and $\mathbf{j}$ electrical switch 


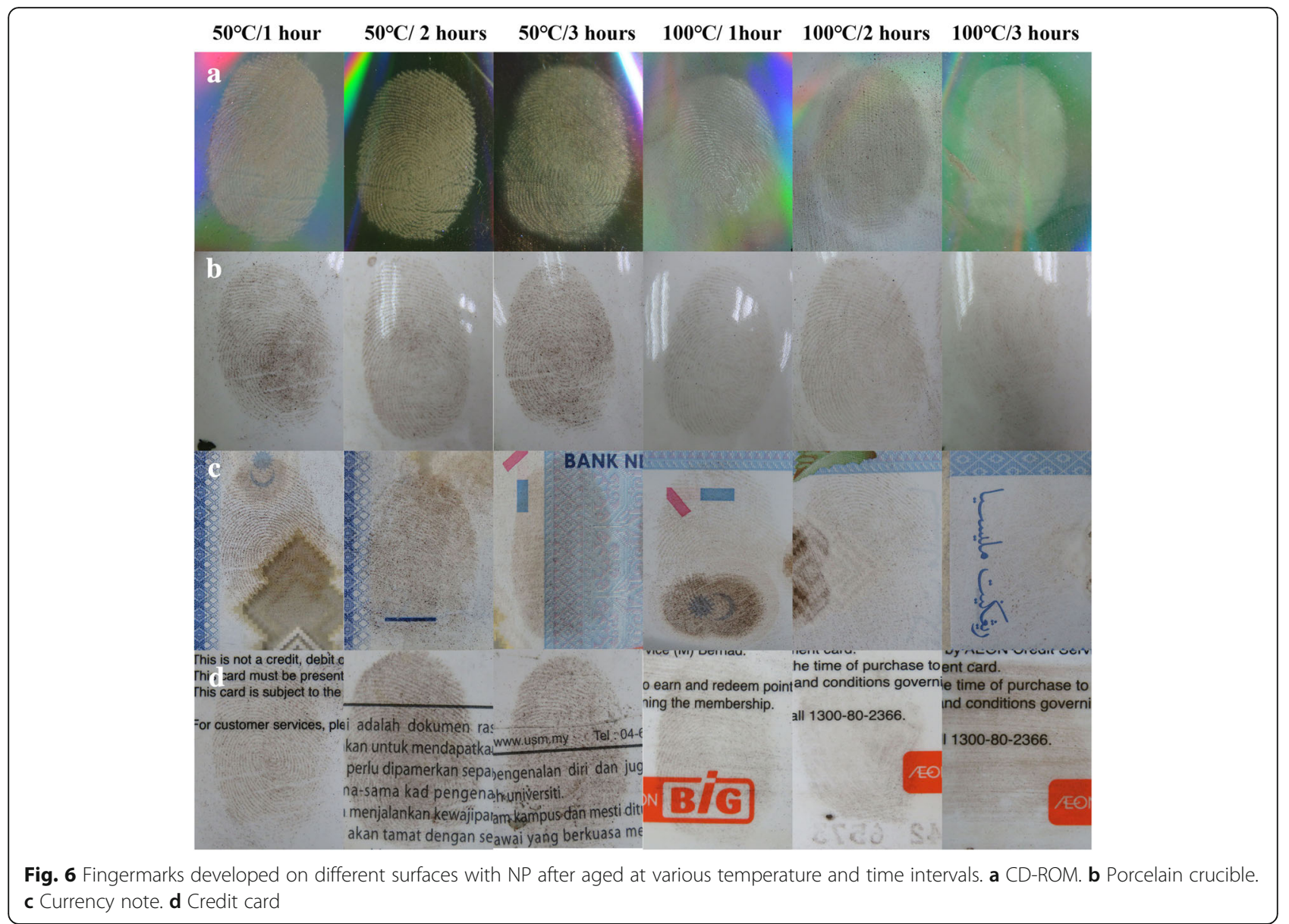

The statistical analyses performed for multiple donors showed insignificant variance for clarity and contrast for fingermarks developed with NP and BP. Nevertheless, the Wilcoxon signed-rank tests performed for depletion study further ratified significant variance results $(p$ value $<0.05)$ for fingermarks developed with both powders, except on surface of porcelain crucible, stainless steel, CD-ROM, and credit card. BP exhibited enhanced performance on tempered glass, gloss painted wood, brown tape, currency note and translucent plastic, while NP had advantage over

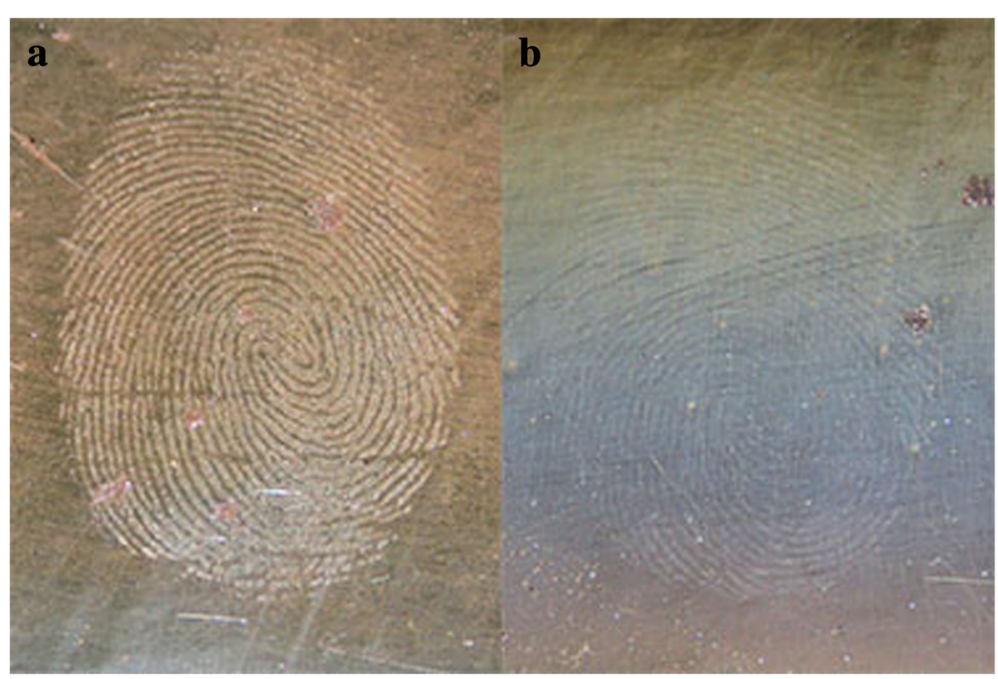

Fig. 7 Fingermarks deposited on stainless steel surface developed with a NP and $\mathbf{b}$ BP. Photographs taken using flash photography 


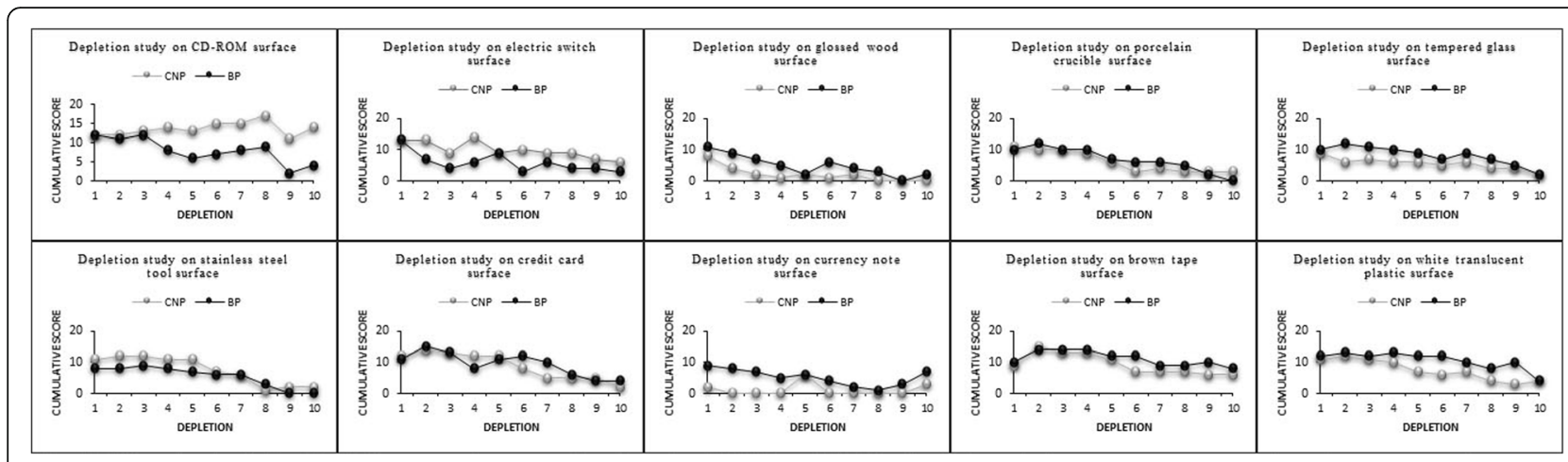

Fig. 8 Line charts that reflect fingermark scores developed with NP and BP for depletion study on non-porous and semi-porous surfaces

the surface of electrical switch. Similarly, ageing studies revealed that BP outperformed NP on several surfaces such as electrical switch, gloss painted wood, credit card and currency note. As such, the statistical analyses displayed significant variance for the efficiency exerted between NP and BP, where BP performed better on majority of the surfaces.

NP synthesised from rice husk in the present study proved to be efficacious for the development of latent fingermarks, deposited on non-porous and semi-porous surfaces. NP was generated as a by-product from the acid digestion of rice husk, a sustainable source, conducted to remove trace metal impurities before extraction of silica. Generally, the filtrate obtained from this process is discarded and becomes waste material. However, it was demonstrated that fluorescent carbon nanoparticles and NP can be extracted from the filtrate simply by ageing. Convective heating process used to govern the nanoparticle growth offers a better control over the formation of the particle, growth rate and agglomeration.

The powder was applied using light strokes of the brush conforming to the commonly used powder dusting technique. Good contrast was visible owing to the dark brown colour of the developed fingermarks on light coloured non-porous surfaces. Appreciable results have been obtained on semi-porous surfaces too. Surfaces selected in present study are generally encountered in the scene of crime. Although powder dusting method is an efficient and simple mean of latent fingermark development, excessive powdering or brushing can lead to the destruction of ridge details necessary for identification. It was observed that BP binds strongly to the fingermark residue as well as finger-mark bearing substrate. On the other hand, NP can be easily removed from the substrate by light brushing along finger-mark ridges.

Commercial BP was made of iron (II, III) oxide nanoparticles and lycopodium, and it was evident that both iron oxide and carbon have non-specific interaction with the fingermark and substrate bearing the fingermark. The black iron oxide is a substance classified under two hazardous class, acute toxicity and self-heating substance. Black iron oxide causes acute toxicity when exposed through skin, inhalation or ingestion. Additionally, lycopodium is classified as flammable solid.

\section{Conclusion}

NP presented in this study had demonstrated sustainable, effective and a green alternative for visualisation of

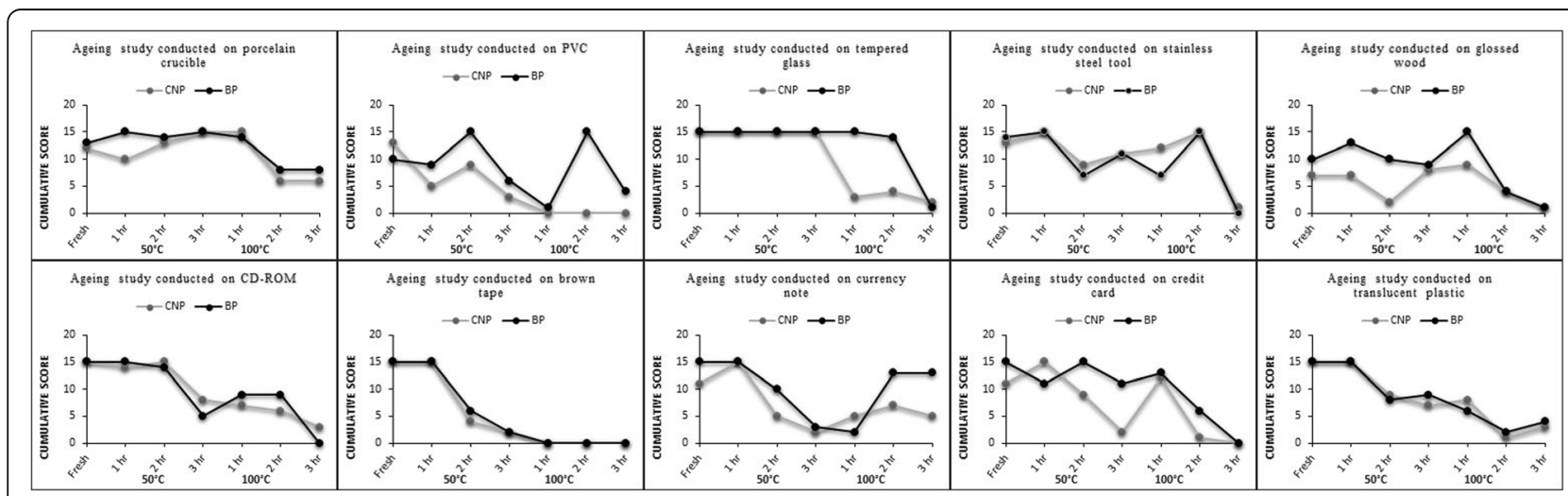

Fig. 9 Line charts that reflect fingermark scores developed with NP and BP for ageing study on non-porous and semi-porous surfaces 
Table 2 Findings of Wilcoxon signed-rank test for scores of fingermarks retrieved from depletion and ageing studies upon each tested surface

\begin{tabular}{|c|c|c|c|c|c|c|c|c|}
\hline Material & Negative rank & Positive rank & Ties & Sig./p value & $Z$ & $N$ & Median $\mathrm{CNP}_{18}$ & Median BP \\
\hline \multicolumn{9}{|c|}{ Multiple donors studies } \\
\hline Porcelain crucible & 6 & 11 & 19 & 0.435 & $-0.776^{b}$ & 36 & 2.5 & 2.0 \\
\hline \multicolumn{9}{|l|}{ Depletion studies } \\
\hline Porcelain crucible & 9 & 8 & 13 & 0.403 & $-0.836^{b}$ & 30 & 2.0 & 2.5 \\
\hline Electrical switch & 4 & 20 & 6 & 0.001 & $-3.229^{c}$ & 30 & 4.0 & 1.5 \\
\hline Tempered glass & 18 & 3 & 9 & 0.001 & $-3.422^{b}$ & 30 & 2.0 & 3.0 \\
\hline Stainless steel tool & 9 & 14 & 7 & 0.076 & $-1.775^{c}$ & 30 & 2.0 & 2.0 \\
\hline Glossed wood & 20 & 2 & 8 & 0.000 & $-3.788^{b}$ & 30 & 0.0 & 1.5 \\
\hline CD-ROM & 7 & 6 & 17 & 0.829 & $-0.216^{c}$ & 30 & 3.0 & 3.0 \\
\hline Brown tape & 17 & 3 & 10 & 0.002 & $-3.144^{b}$ & 30 & 3.0 & 4.0 \\
\hline Credit card & 14 & 5 & 11 & 0.340 & $-0.955^{b}$ & 30 & 3.0 & 3.0 \\
\hline Currency note & 24 & 1 & 5 & 0.000 & $-4.274^{b}$ & 30 & 0.0 & 2.0 \\
\hline Translucent plastic & 20 & 1 & 9 & 0.000 & $-3.886^{\mathrm{b}}$ & 30 & 2.5 & 4.0 \\
\hline \multicolumn{9}{|l|}{ Ageing studies } \\
\hline Porcelain crucible & 8 & 4 & 9 & 0.266 & $-1.112^{b}$ & 21 & 4.0 & 5.0 \\
\hline Electrical switch & 15 & 2 & 4 & 0.003 & $-2.960^{\mathrm{b}}$ & 21 & 1.0 & 3.0 \\
\hline Tempered glass & 6 & 1 & 14 & 0.031 & $-2.156^{b}$ & 21 & 5.0 & 5.0 \\
\hline Stainless steel tool & 1 & 6 & 14 & 0.058 & $-1.897^{c}$ & 21 & 4.0 & 4.0 \\
\hline Glossed wood & 12 & 3 & 6 & 0.002 & $-3.041^{b}$ & 21 & 2.0 & 3.0 \\
\hline CD-ROM & 5 & 8 & 8 & 0.740 & $-0.332^{c}$ & 21 & 4.0 & 4.0 \\
\hline Brown tape & 3 & 1 & 17 & 0.317 & $-1.000^{b}$ & 21 & 1.0 & 1.0 \\
\hline Credit card & 11 & 2 & 8 & 0.015 & $-2.433^{b}$ & 21 & 3.0 & 4.0 \\
\hline Currency note & 11 & 2 & 8 & 0.020 & $-2.331^{b}$ & 21 & 2.0 & 4.0 \\
\hline Translucent plastic & 6 & 5 & 10 & 0.891 & $-0.137^{b}$ & 21 & 3.0 & 2.0 \\
\hline
\end{tabular}

${ }^{a}$ Negative ranks: $\mathrm{CNP}_{18}<\mathrm{BP}$

${ }^{b}$ Positive ranks: $\mathrm{CNP}_{18}>\mathrm{BP}$

${ }^{\mathrm{C}}$ Ties: $\mathrm{CNP}_{18}=\mathrm{BP}$

latent fingermarks deposited varied non-porous and semi-porous surfaces. The results depict that the NP offers a selective interaction with fingermark residue, and thus offer clear and sharp images of fingermark ridges. In addition, the NP has been proven to outweigh BP due to two reasons: (1) the easy synthesis method using rice husk, an agricultural waste, and (2) its low toxicity content in the powder. On top of that, rice husk, which is a sustainable source, is available in abundance and the use of this rice husk in such a way decreases the accumulating wastes. This method also recycles the acid digestion residue that is usually discarded during the extraction of silica from rice husk. In addition, the method proposed does not require intense energy or costly components, thus suggesting the feature of being environmentally friendly, apart from being cost-effective. Further studies on larger scale with more surfaces and comparison with other available powders would be needed to establish the superiority and applicability of NP in routine forensic casework.

\section{Abbreviations}

BP: Black powder; EDX: Energy-dispersive spectroscopy; FTIR: Fourier-transform infrared spectroscopy; NP: Nanocarbon powder; SEM: Scanning electron microscopy; XRD: X-ray diffraction spectroscopy

\section{Acknowledgements}

Not applicable.

Funding

Financial support for this research by the Universiti Sains Malaysia (USM) Research Grant 1001/PPSK/812125.

Availability of data and materials

Please contact author for data requests.

Author's contributions

All authors contributed to the design of the study. RR performed the experiments. RR and NF analysed the data. All authors discussed and wrote the manuscript. All authors read and approved the final manuscript

\section{Author's information}

Not applicable.

\section{Ethics approval and consent to participate}

The human ethical clearance was approved by Human Research Ethics Committee, Universiti Sains Malaysia (reference number: USM/JEPeM/280.5 (1.3)). 


\section{Consent for publication}

Consent to publish was obtained from each participant.

\section{Competing interests}

The authors declare that they have no competing interests.

\section{Publisher's Note}

Springer Nature remains neutral with regard to jurisdictional claims in published maps and institutional affiliations.

\section{Author details}

${ }^{1}$ Forensic Science Programme, School of Health Sciences, Universiti Sains Malaysia, Health Campus, 16150 Kubang Kerian, Kelantan, Malaysia. ${ }^{2}$ Biomedical Science Programme, School of Health Sciences, Universiti Sains Malaysia, Health Campus, 16150 Kubang Kerian, Kelantan, Malaysia.

\section{Received: 11 July 2018 Accepted: 25 October 2018}

\section{Published online: 07 November 2018}

\section{References}

Balasooriya ER, Jayasinghe CD, Jayawardena UA et al (2017) Honey mediated green synthesis of nanoparticles: new era of safe nanotechnology. J Nanomater 2017:1-10

Becue A, Moret S, Champod C, Margot P (2011) Use of stains to detect fingermarks. Inf Heal 86:140-160

Branca C, Frusteri F, Magazu V, Mangione A (2004) Characterization of carbon nanotubes by TEM and infrared spectroscopy. J Phys Chem B 108:3469-3473

Cao L, Wang X, Meziani MJ et al (2007) Carbon dots for multiphoton bioimaging carbon dots for multiphoton bioimaging. J Am Chem Soc 129:11318-11319

Chadwick S, Maynard P, Kirkbride P et al (2012) Styryl dye coated metal oxide powders for the detection of latent fingermarks on non-porous surfaces. Forensic Sci Int 219:208-214

Champod C, Lennard C, Margot P, Stoilovic M (2004) Fingerprints and other ridge skin impressions, first edit. CRC Press, USA

Choi MJ, McBean KE, Ng PHR et al (2008) An evaluation of nanostructured zinc oxide as a fluorescent powder for fingerprint detection. J Mater Sci 43:732-737

Cohen Y, Rozen E, Azoury M et al (2012) Survivability of latent fingerprints part I: adhesion of latent fingerprints to smooth surfaces. J Forensic Identif 62:47-53

Dhall JK, Kapoor AK (2016) Development of latent prints exposed to destructive crime scene conditions using wet powder suspensions. Egypt J Forensic Sci 6:396-404

Fan RJ, Sun Q, Zhang L et al (2014) Photoluminescent carbon dots directly derived from polyethylene glycol and their application for cellular imaging. Carbon N Y 71:87-93

Fieldhouse S, Oravcova E, Walton-Williams L (2016) The effect of DNA recovery on the subsequent quality of latent fingermarks. Forensic Sci Int 267:78-88

Garrigue P, Delville M-H, Labrugere C et al (2004) Top - down approach for the preparation of colloidal carbon nanoparticles. Chem Mater 16:2984-2986

Goode GC, Morris JR (1983) Latent fingerprints: a review of their origin, composition and methods for detection. Forensic Sci Int 25:1-137

Han L, Ghosh D, Chen W et al (2009) Nanosized carbon particles from natural gas soot. Chem Mater 21:2803-2809

Hu BB, Wang K, Wu L et al (2010) Engineering carbon materials from the hydrothermal carbonization process of biomass. Adv Mater 22:813-828

Hu S-L, Niu K-Y, Sun J et al (2009) One-step synthesis of fluorescent carbon nanoparticles by laser irradiation. J Mater Chem 19:484-488

Jelly R, Patton ELT, Lennard C et al (2009) The detection of latent fingermarks on porous surfaces using amino acid sensitive reagents: a review. Anal Chim Acta 652:128-142

Lee HC, Gaensslen RE (2012) Lee and Gaensslen's: advances in fingerprint technology, 3rd edit. CRC press, Boca Raton, Florida

Lee JH, Kwon JH, Lee J-W et al (2017) Preparation of high purity silica originated from rice husks by chemically removing metallic impurities. J Ind Eng Chem 50:79-85

Li H, He X, Liu Y et al (2011a) One-step ultrasonic synthesis of water-soluble carbon nanoparticles with excellent photoluminescent properties. Carbon N Y 49:605-609

Li H, He X, Liu Y et al (2011b) Synthesis of fluorescent carbon nanoparticles directly from active carbon via a one-step ultrasonic treatment. Mater Res Bull 46:147-151
Liu Y, Guo Y, Gao W et al (2012) Simultaneous preparation of silica and activated carbon from rice husk ash. J Clean Prod 32:204-209. https://doi.org/10.1016/j. jclepro.2012.03.021

Luduena L, Fasce D, Alvarez VA, Stefani PM (2011) Nanocellulose from rice husk following alkaline treatment to remove silica. BioResources 6:1440-1453

Meiling TT, Cywiński PJ, Bald I (2016) White carbon: fluorescent carbon nanoparticles with tunable quantum yield in a reproducible green synthesis. Sci Rep 6:28557

Ngu PZZ, Chia SPP, Fong JFY, Ng SM (2016) Synthesis of carbon nanoparticles from waste rice husk used for the optical sensing of metal ions. New Carbon Mater 31:135-143

Nithin MD, Balaraj BM, Manjunatha B, Mestri SC (2009) Study of fingerprint classification and their gender distribution among south Indian population. J Forensic Legal Med 16:460-463

Peng H, Travas-Sejdic J (2009) Simple aqueous solution route to luminescent carbogenic dots from carbohydrates. Chem Mater Commun 21:5563-5565

Rajan R, Zakaria Y, Shamsuddin S, Hassan NFN (2018) Synthesis of carbon nanoparticle from Rice husk by acid digestion for fingermark dusting application. Malaysian J Forensic Sci 8:7-13

Ray SC, Saha A, Jana NR, Sarkar R (2009) Fluorescent carbon nanoparticles: synthesis, characterization, and bioimaging application. J Phys Chem C 113: 18546-18551

Roshni V, Ottoor D (2015) Synthesis of carbon nanoparticles using one step green approach and their application as mercuric ion sensor. J Lumin 161:117-122

Sears VG, Bleay SM, Bandey HL, Bowman VJ (2012) A methodology for finger mark research. Sci Justice 52:145-160

Shen Y (2017) Rice husk silica derived nanomaterials for battery applications - a literature review. J Agric Food Chem 65:995-1004

Shen Y, Zhao P, Shao Q (2014) Porous silica and carbon derived materials from rice husk pyrolysis char. Microporous Mesoporous Mater 188:46-76

Sodhi GS, Kaur J (2001) Powder method for detecting latent fingerprints: a review. Forensic Sci Int 120:172-176

Song X, Zhang Y, Chang C (2012) Novel method for preparing activated carbons with high specific surface area from rice husk. Ind Eng Chem Res 51:15075-15081

Sun X, Li Y (2004) Colloidal carbon spheres and their core/shell structures with noble-metal nanoparticles. Angew Chem Int Ed 43:597-601

Sun YP, Zhou B, Lin Y et al (2006) Quantum-sized carbon dots for bright and colorful photoluminescence. J Am Chem Soc 128:7756-7757

Vaibhav V, Vijayalakshmi U, Roopan SM, Mohana Roopan S (2015) Agricultural waste as a source for the production of silica nanoparticles. Spectrochim Acta - Part A Mol Biomol Spectrosc 139:515-520

Wang L, Guo Y, Zhu Y et al (2010) A new route for preparation of hydrochars from rice husk. Bioresour Technol 101:9807-9810

Wang Y, Hu A (2014) Carbon quantum dots: synthesis, properties and applications. J Mater Chem C 2:6921-6939

Wilshire B (1996) Advances in fingerprint detection. Endeavour 20:12-15

Wu L, Cai X, Nelson K et al (2013) A green synthesis of carbon nanoparticle from honey for real- time photoacoustic imaging. Nano Res 6:312-325

Xu X, Ray R, Gu Y et al (2004) Electrophoretic analysis and purification of fluorescent single-walled carbon nanotube fragments. J Am Chem Soc 126: 12736-12737

Yamashita B, French M (2011) Latent print development. In: Holder EH, Robinson LO, Laub JH (eds) The fingerprint sourcebook, 1st Editio. U.S. Department of justice, Office of Justice Programs, National Institute of Justice., Washington, DC, p 7.1-7.67

Yu C, Xuan T, Chen Y et al (2015) A facile, green synthesis of highly fluorescent carbon nanoparticles from oatmeal for cell imaging. J Mater Chem C 3:9514-9518

Zhao Q-L, Zhang Z-L, Huang B-H et al (2008) Facile preparation of low cytotoxicity fluorescent carbon nanocrystals by electrooxidation of graphite. Chem Commun 7:5116-5118

Zhu H, Wang X, Li Y et al (2009) Microwave synthesis of fluorescent carbon nanoparticles with electrochemiluminescence properties. Chem Commun (34):5118-5120 